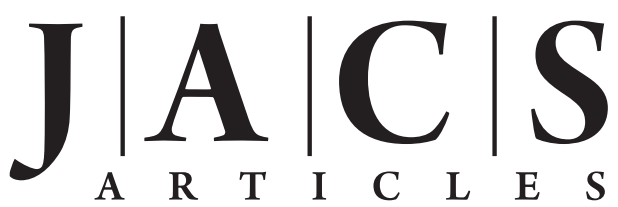

Published on Web 12/14/2009

\title{
Orthogonal Orientation Control of Carbon Nanotube Growth
}

\author{
Weiwei Zhou, Lei Ding, Sungwoo Yang, and Jie Liu* \\ Department of Chemistry, Duke University, Durham, North Carolina 27708 \\ Received October 9, 2009; E-mail: j.liu@ duke.edu
}

\begin{abstract}
Carbon nanotubes (CNTs) have attracted attention for their remarkable electrical properties and have being explored as one of the best building blocks in nano-electronics. A key challenge to realize such potential is the control of the nanotube growth directions. Even though both vertical growth and controlled horizontal growth of carbon nanotubes have been realized before, the growth of complex nanotube structures with both vertical and horizontal orientation control on the same substrate has never been achieved. Here, we report a method to grow three-dimensional (3D) complex nanotube structures made of vertical nanotube forests and horizontal nanotube arrays on a single substrate and from the same catalyst pattern by an orthogonally directed nanotube growth method using chemical vapor deposition (CVD). More importantly, such a capability represents a major advance in controlled growth of carbon nanotubes. It enables researchers to control the growth directions of nanotubes by simply changing the reaction conditions. The high degree of control represented in these experiments will surely make the fabrication of complex nanotube devices a possibility.
\end{abstract}

\section{Introduction}

Controlling the orientation of nanotubes on a substrate is a major challenge in the large-scale fabrication of nanotube-based devices. ${ }^{1-4}$ In recent years major breakthroughs have been achieved in the directed growth of aligned nanotubes on substrates $^{5-7,9-11,12,13-15}$ and post-synthesis self-assembly of

(1) Kang, S. J.; Kocabas, C.; Ozel, T.; Shim, M.; Pimparkar, N.; Alam, M. A.; Rotkin, S. V.; Rogers, J. A. Nat. Nanotechnol. 2007, 2, 230 236.

(2) Kocabas, C.; Dunham, S.; Cao, Q.; Cimino, K.; Ho, X. N.; Kim, H. S.; Dawson, D.; Payne, J.; Stuenkel, M.; Zhang, H.; Banks, T.; Feng, M.; Rotkin, S. V.; Rogers, J. A. Nano Lett. 2009, 9, 1937-1943.

(3) Ryu, K.; Badmaev, A.; Wang, C.; Lin, A.; Patil, N.; Gomez, L.; Kumar, A.; Mitra, S.; Wong, H. S. P.; Zhou, C. W. Nano Lett. 2009, 9, 189197.

(4) Ishikawa, F. N.; Chang, H. K.; Ryu, K.; Chen, P. C.; Badmaev, A.; De Arco, L. G.; Shen, G. Z.; Zhou, C. W. ACS Nano 2009, 3, 73-79.

(5) Fan, S. S.; Chapline, M. G.; Franklin, N. R.; Tombler, T. W.; Cassell, A. M.; Dai, H. J. Science 1999, 283, 512-514.

(6) Hata, K.; Futaba, D. N.; Mizuno, K.; Namai, T.; Yumura, M.; Iijima, S. Science 2004, 306, 1362-1364.

(7) Zhang, G. Y.; Mann, D.; Zhang, L.; Javey, A.; Li, Y. M.; Yenilmez, E.; Wang, Q.; McVittie, J. P.; Nishi, Y.; Gibbons, J.; Dai, H. J. Proc. Natl. Acad. Sci. U.S.A. 2005, 102, 16141-16145.

(8) Murakami, Y.; Chiashi, S.; Miyauchi, Y.; Hu, M. H.; Ogura, M.; Okubo, T.; Maruyama, S. Chem. Phys. Lett. 2004, 385, 298-303.

(9) Zhang, Y. G.; Chang, A. L.; Cao, J.; Wang, Q.; Kim, W.; Li, Y. M.; Morris, N.; Yenilmez, E.; Kong, J.; Dai, H. J. Appl. Phys. Lett. 2001, 79, 3155-3157.

(10) Huang, S. M.; Cai, X. Y.; Liu, J. J. Am. Chem. Soc. 2003, 125, 56365637.

(11) Ismach, A.; Segev, L.; Wachtel, E.; Joselevich, E. Angew. Chem., Int. Ed. 2004, 43, 6140-6143.

(12) Han, S.; Liu, X. L.; Zhou, C. W. J. Am. Chem. Soc. 2005, 127, 5294 5295.

(13) Kocabas, C.; Hur, S. H.; Gaur, A.; Meitl, M. A.; Shim, M.; Rogers, J. A. Small 2005, 1, 1110-1116.

(14) Zhou, W. W.; Han, Z. Y.; Wang, J. Y.; Zhang, Y.; Jin, Z.; Sun, X.; Zhang, Y. W.; Yan, C. H.; Li, Y. Nano Lett. 2006, 6, 2987-2990.

(15) Ding, L.; Tselev, A.; Wang, J. Y.; Yuan, D. N.; Chu, H. B.; McNicholas, T. P.; Li, Y.; Liu, J. Nano Lett. 2009, 9, 800-805. nanotubes onto silicon wafers. ${ }^{16-18}$ For example, horizontally aligned arrays of single-walled carbon nanotubes (SWNTs) with high density were successfully synthesized on single-crystal quartz wafers by the guidance of surface lattices, and SWNTbased thin film field effective transistors (FETs) and simple logic circuits were fabricated in a wafer scale. ${ }^{1,3}$ Furthermore, more complex structures of nanotubes have been reported, which include two-dimensional cross networks of SWNTs in $x-y$ plane ${ }^{19-21}$ and three-dimensional (3D) micromechanical systems based on nanotube forests. ${ }^{22}$ Horizontal nanotube arrays and vertical nanotube forests are both important building blocks in nanodevices. ${ }^{23,24}$ However, there is no report on effectively assembling both the horizontal nanotube arrays and vertical nanotube forests on the same substrate into a 3D nanotube structures. Such capability is important for creating complex nanotube structures in highly integrated circuits.

Herein, we present an orthogonally directed nanotube growth method to create new 3D nanotube structures made of both vertical forests and horizontal arrays of carbon nanotubes on a single substrate and from the same catalyst pattern. In order to achieve well-controlled 3D nanotube structures, a substrate that can provide control of both horizontal and vertical nanotube growth direction is critical. In the past few years, superlong

(16) Rao, S. G.; Huang, L.; Setyawan, W.; Hong, S. H. Nature 2003, 425, $36-37$.

(17) Li, X. L.; Zhang, L.; Wang, X. R.; Shimoyama, I.; Sun, X. M.; Seo, W. S.; Dai, H. J. J. Am. Chem. Soc. 2007, 129, 4890-4891.

(18) LeMieux, M. C.; Roberts, M.; Barman, S.; Jin, Y. W.; Kim, J. M.; Bao, Z. N. Science 2008, 321, 101-104.

(19) Huang, S. M.; Maynor, B.; Cai, X. Y.; Liu, J. Adv. Mater. 2003, 15, 1651-1655.

(20) Ismach, A.; Joselevich, E. Nano Lett. 2006, 6, 1706-1710.

(21) Zhou, W. W.; Ding, L.; Liu, J. Nano Res. 2009, 2, 593-598.

(22) Hayamizu, Y.; Yamada, T.; Mizuno, K.; Davis, R. C.; Futaba, D. N.; Yumura, M.; Hata, K. Nat. Nanotechnol. 2008, 3, 289-294.

(23) Graham, A. P.; Duesberg, G. S.; Seidel, R. V.; Liebau, M.; Unger, E.; Pamler, W.; Kreupl, F.; Hoenlein, W. Small 2005, 1, 382-390.

(24) Robertson, J. Mater. Today 2007, 10, 36-43. 
forests of nanotubes in silicon wafers were synthesized by waterassisted chemical vapor deposition (CVD). ${ }^{6,25-29}$ An important "trick" in the synthesis is the introduction of a thin intermediate layer of $\mathrm{Al}_{2} \mathrm{O}_{3}$ between the catalyst and the substrate to increase the efficiency of growth. However, the amorphous nature of the intermediate layer makes the control of the horizontal growth direction difficult. We found that on quartz substrate there is no need for such an adhesion layer for the growth of highquality nanotube forests. Additionally, certain quartz substrates, Y-cut, ST-cut, etc., have been used to grow high-quality horizontally aligned nanotubes. ${ }^{13,15,30-32}$ In our experiments, catalysts were patterned on a quartz surface by a one-step photolithography method. ${ }^{33}$ The catalyst pattern can be used to catalyze the growth of a nanotube forest composed of fewwalled carbon nanotubes (FWNTs) with 2-4 walls by an enhancer-assisted CVD as well as the horizontally aligned arrays of SWNTs by a methanol/ethanol CVD process. Complex 3D nanotube structures can be easily generated, and based on this 3D nanotube structure, all-carbon FET devices can be fabricated using the forest islands as contact electrodes and horizontal arrays as FET channels without the need for metal electrodes. This complex architecture of nanotubes enables the further application of nanotubes in highly integrated dc circuits, MEMS, and RF devices.

\section{Experimental Methods}

Fabrication of Catalyst Patterns on Quartz, Sapphire, and Silicon Wafers. ST-cut quartz wafers ( $36^{\circ}$ Y-cut, University Wafer) and C-plane sapphire wafers (Hoffman Materials Inc.) were first annealed at $850{ }^{\circ} \mathrm{C}$ in $\mathrm{Ar}$ for $30 \mathrm{~min}$. Shipley 1813 positive photoresist (PR) (Shipley Company) mixed with $\mathrm{FeCl}_{3}$ (purity $97 \%$, Sigma-Aldrich) at the concentration of $2-10 \mathrm{mM}$ was used as the catalyst precursor. Standard photolithography was used for patterning catalysts on quartz, sapphire, and silicon wafers (Silicon Quest International, Inc.). The PR was spin-coated onto substrates at a speed of $1500 \mathrm{rpm}$ and subsequently baked for $100-150 \mathrm{~s}$ on a hot plate at $115^{\circ} \mathrm{C}$. Suss MicroTec MJB3 with the light intensity of $9.2 \mathrm{~mW}$ was used for the exposure and exposure time is around 11s. After exposure, wafers were soaked into MF-319 developer for about $45 \mathrm{~s}$ and then immediately rinsed with plenty of water and blow-dried with $\mathrm{N}_{2}$.

Growth of Vertical FWNT Forests on Quartz. Wafers with catalyst patterns were put into the center of a Lindberg $1 \mathrm{in}$. furnace and were calcined at $700{ }^{\circ} \mathrm{C}$ in air for $10 \mathrm{~min}$ in order to remove the PR layer and form $\mathrm{Fe}_{x} \mathrm{O}_{y}$ catalyst particles. After cooling to room temperature, the furnace was reheated to $850{ }^{\circ} \mathrm{C}$ under the protection of Ar. The wafers were located 3 in. downstream from the center of furnace. For a typical CVD growth of nanotube forests,

(25) Futaba, D. N.; Hata, K.; Namai, T.; Yamada, T.; Mizuno, K.; Hayamizu, Y.; Yumura, M.; Iijima, S. J. Phys. Chem. B 2006, 110, 8035-8038.

(26) Nishino, H.; Yasuda, S.; Namai, T.; Futaba, D. N.; Yamada, T.; Yumura, M.; Iijima, S.; Hata, K. J. Phys. Chem. C 2007, 111, 1796117965.

(27) Sugime, H.; Noda, S.; Maruyama, S.; Yamaguchi, Y. Carbon 2009, 47, 234-241.

(28) Yamada, T.; Namai, T.; Hata, K.; Futaba, D. N.; Mizuno, K.; Fan, J.; Yudasaka, M.; Yumura, M.; Iijima, S. Nat. Nanotechnol. 2006, 1, 131136.

(29) Yamada, T.; Maigne, A.; Yudasaka, M.; Mizuno, K.; Futaba, D. N.; Yumura, M.; Iijima, S.; Hata, K. Nano Lett. 2008, 8, 4288-4292.

(30) Kocabas, C.; Shim, M.; Rogers, J. A. J. Am. Chem. Soc. 2006, 128 4540-4541.

(31) Ding, L.; Yuan, D. N.; Liu, J. J. Am. Chem. Soc. 2008, 130, 54285429.

(32) Yuan, D. N.; Ding, L.; Chu, H. B.; Feng, Y. Y.; McNicholas, T. P.; Liu, J. Nano Lett. 2008, 8, 2576-2579.

(33) Zhou, W. W.; Rutherglen, C.; Burke, P. Nano Res. 2008, 1, 158-165.

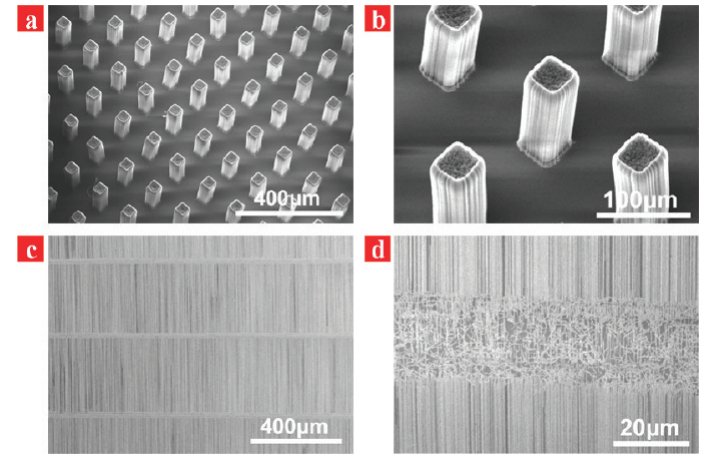

Figure 1. SEM images of selective growth of vertical aligned islands of CNTs (a, b) and horizontal aligned arrays of CNTs (c, d) on quartz surfaces. (b and d) Higher-magnification SEM images of (a and c), respectively.

a flow of $\mathrm{C}_{2} \mathrm{H}_{4}(90 \mathrm{sccm})$ and $\mathrm{H}_{2}(200 \mathrm{sccm})$ together with an $\mathrm{Ar} /$ water vapor mixture $(800 \mathrm{sccm})$, obtained by passing the Ar gas through a water bubbler, was introduced into the system at the furnace temperature of $850{ }^{\circ} \mathrm{C}$ for $10 \mathrm{~min}$. For lower growth temperatures, the concentration of water vapor in furnace is lower by decreasing the flow rate of Ar. For example, at $800{ }^{\circ} \mathrm{C}$, the optimal flow rate of Ar/water vapor mixture is $500 \mathrm{sccm}$.

Growth of Horizontal SWNT Arrays on Quartz. After the same thermal treatment for removing PR, the substrates were heated up to $800{ }^{\circ} \mathrm{C}$ and kept there for $15 \mathrm{~min}$ with a flow of hydrogen (750 sccm), followed by CVD growth of SWNTs at $900{ }^{\circ} \mathrm{C}$ with a flow of hydrogen $(750 \mathrm{sccm})$ and argon $(600 \mathrm{sccm}$, through a methanol bubbler, and $150 \mathrm{sccm}$, through an ethanol bubbler) for $15 \mathrm{~min}$. In the process of growing 3D CNT architectures after the growth of vertical FWNT forests, the flow of hydrogen $(750 \mathrm{sccm})$ was immediately introduced. There is no need to do thermal treatment for removing PR anymore.

Fabrication of FET Devices with Top-Gated Configuration. The gate material was made from a solid polymer electrolyte, poly(ethylene oxide)/lithium perchlorate mixture $\left(\mathrm{PEO}: \mathrm{LiClO}_{4} \cdot 3 \mathrm{H}_{2} \mathrm{O}\right)$. PEO (MW 100 000) and $\mathrm{LiClO}_{4} \cdot 3 \mathrm{H}_{2} \mathrm{O}$ were from Aldrich Chemical Co., Inc. The polymer-to-perchlorate weight ratio was 1:0.18. The methanol solution was centrifuged to remove undisolved particles of PEO from the precursor. The supernatant was drop dried on a chip with patterned electrodes. The chip was heated to $45^{\circ} \mathrm{C}$ during the precursor deposition. The liquid was allowed to dry, and then the samples were put into vacuum for several hours to remove residual solvent and moisture. Keithley 4200-SCS semiconductor characterization system was used for measuring the electrical characteristics.

\section{Results and Discussion}

Orthogonally Directed Growth of Nanotubes on Quartz Substrates. Quartz substrate has been demonstrated to guide the growth of densely aligned SWNT by a few groups. ${ }^{13,15,31-34}$ Especially according to our recent results, the density of SWNT arrays is as high as $\sim 20 \mathrm{SWNTs} / \mu \mathrm{m},{ }^{35}$ which has never been achieved by using silicon wafer or single-crystal sapphire. It indicates that single-crystal quartz is an efficient substrate for the growth of a horizontally aligned nanotube. Additionally, the substrate can be used to grow a vertical nanotube forest when the growth conditions are altered. SEM images $a$ and $b$ of Figure 1 show well-ordered, vertically aligned structures of vertical nanotube islands on quartz wafers which were synthesized using patterned Shipley $1813 / \mathrm{FeCl}_{3}$ catalyst precursors and water-

(34) Kocabas, C.; Kang, S. J.; Ozel, T.; Shim, M.; Rogers, J. A. J. Phys. Chem. C 2007, 111, 17879-17886.

(35) Ding, L.; Zhou, W.; Thomas, P. M.; Wang, J.; Chu, H.; Li, Y.; Liu, J. Nano Res. 2009, 2, 903-910. 


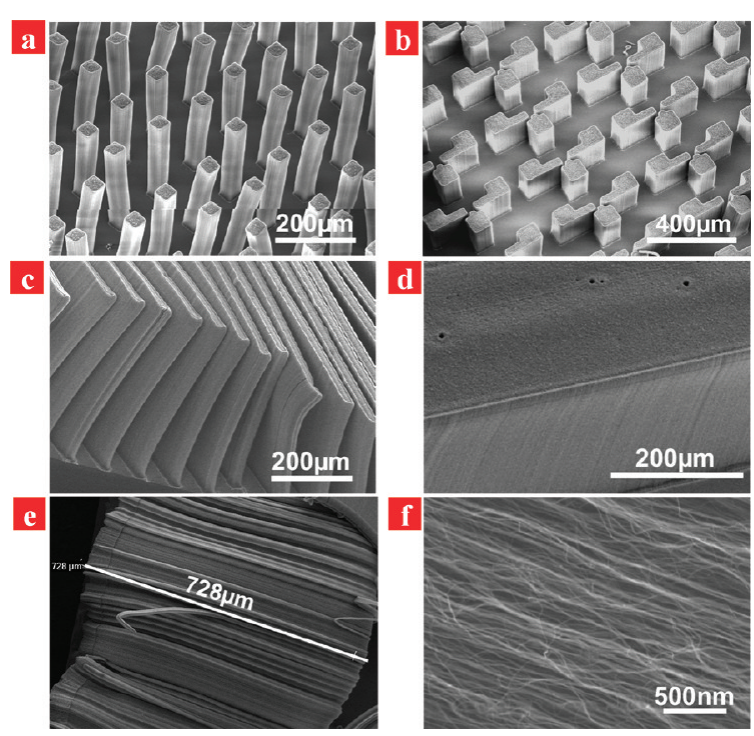

Figure 2. SEM images of various organized CNT forests on quartz wafers by water-assisted ethylene CVD. SEM image of (a) cubic pillars, (b) CNT electrodes, (c) CNT walls, (d) CNT forest without patterns, (e) CNT forest with the height of $728 \mu \mathrm{m}$. (f) Higher-magnification SEM image of CNT forests.

assisted ethylene-CVD method at $850{ }^{\circ} \mathrm{C}$. Using the same catalyst precursors and a methanol/ethanol CVD process at 900 ${ }^{\circ} \mathrm{C}$, densely aligned arrays of horizontal nanotubes can grow from catalyst patterns (shown in Figure 1c and 1d), of which the orientation of is [2110] direction of the Y-cut quartz. ${ }^{34}$ This result illuminated that single-crystal quartz not only can guide the growth of dense horizontal nanotubes on the surface, but also support the growth of vertical nanotubes in the $\mathrm{Z}$ directions.

Most interestingly, we noticed that we can control the growth parameters to ensure almost no horizontal nanotube growth when growing vertical nanotube forests and no vertical nanotube growth when growing horizontal nanotube arrays (Figure $1 \mathrm{~b}$ and Figure 1d). In our experiments, nanotube forests can be easily synthesized within a wide range of the furnace temperature from 800 to $850{ }^{\circ} \mathrm{C}$ by using different optimal concentrations of enhancers (Figure $1 \mathrm{~S}$ in the Supporting Information). Water, acetone, or methanol were found to effectively increase the growth yield of vertical nanotubes as the growth enhancers (Figure $2 \mathrm{~S}$ in the Supporting Information). Meanwhile, the growth of horizontal nanotubes requires a higher temperature $\left(900{ }^{\circ} \mathrm{C}\right)$. Therefore, at the optimized CVD conditions, either vertical or horizontal nanotubes could be selectively synthesized. This orthogonally anisotropic growth of nanotubes on quartz substrates can be realized by controlling the CVD conditions.

We also attempted to find CVD conditions that could simultaneously grow both vertical and horizontal nanotubes. However, the results show that the alignment of horizontal nanotubes and the height of vertical nanotubes both suffer (Figure $3 \mathrm{~S}$ in the Supporting Information). We believe it is because the vertical and horizontal nanotubes each need different carbon feeding rates for growth, and it is difficult to grow both of them at a fixed carbon feeding rate and still achieve highyield and high-quality nanotubes.

Characterizations of Vertical Nanotube Forests and Horizontal Nanotube Arrays. By directly patterning Shipley $1813 / \mathrm{FeCl}_{3}$ catalyst precursors on a quartz surface, various forest patterns of nanotubes were synthesized at wafer scale (Figure $2 a-c$ ). The heights of the vertical forests can be tuned from tens of micrometers to $0.7 \mathrm{~mm}$ (Figure 2e) by changing the CVD conditions. The nanotube forests are free of amorphous carbon and catalyst particles observed in the higher-magnification scanning electron microscope (SEM) image (Figure 2f) and high-resolution transmission electron microscope (HRTEM) image (Figure 3a). In Raman spectra of forests (Figure 3b), there is a relative high-intensity $\mathrm{D}$ band. The radial breathing mode (RBM) peak was seldom detected and is not shown in Figure $3 \mathrm{~b}$. The results of Raman spectroscopy indicate that multiwalled carbon nanotubes are synthesized. According to the data of HRTEM characterization (inset of Figure 3a), the number distribution of nanotube walls is from 2 to 4 , and the diameter distribution is from 4 to $7 \mathrm{~nm}$ with the average diameter of 5.2 $\mathrm{nm}$, which means that vertical forests are made of FWNTs.

On the other hand, horizontal arrays of CNTs are made of SWNTs with the diameters of 1.4-2.0 nm according to AFM and HRTEM data (Figure 3c). The RBM peak, often detected at around $150 \mathrm{~cm}^{-1}$, and the very low-intensity D band in Raman spectra of horizontal CNTs (Figure 3d) indicate that predominately semiconducting SWNTs were synthesized, similar to our earlier results ${ }^{31}$ for the selective growth of high-percentage semiconducting SWNT arrays.

Building a 3D Hierarchical Nanotube Structure. The wellcontrolled CVD growth of nanotubes in two orthogonal directions ensures the synthesis of high-quality nanotubes in both directions, and provides us an approach to construct a more complex structure of nanotubes by combining vertical FWNTs and horizontal SWNTs. We did two separate CVD processes to grow vertical FWNT forests first and then grow horizontal SWNT arrays on the same substrate. The strategy is shown in Scheme 1. SEM images (Figure 4a-e) display a new 3D hierarchical nanotube structure made of orthogonally vertical and horizontal nanotubes which connect between nanotube forest islands on the quartz surface. Horizontal nanotubes show parallel alignment similar to the result of a previous alcohol CVD growth $^{15,31}$ (Figure 1c and d). The RBM peak and the low peak intensity ratio of the D-band over that of the G-band in the Raman spectrum of surface nanotubes (shown in Figure 4f) further verify that SWNTs were synthesized. The results indicate that the existence of vertical FWNT forests in the first step of ethylene CVD has no obvious influence on alignment, orientation, and defect and wall numbers of horizontal nanotubes synthesized in the second step of alcohol CVD. Just by controlling the CVD conditions, we realized the directed growth of aligned nanotubes in both $Z$ direction and $X-Y$ horizontal plane on the same substrate.

Role of Quartz Substrates on the Orthogonally Directed CVD Growth. In order to further investigate the role of quartz substrates, we compared the experimental results obtained on quartz, sapphire, and silicon wafers under the same CVD conditions. We changed the growth temperatures from 750 to $850{ }^{\circ} \mathrm{C}$. The results showed that it is easy to grow FWNT forest on quartz with the height of hundreds of micrometers when the growth temperature is between 800 to $850{ }^{\circ} \mathrm{C}$. On sapphire substrates, there is no success in the growth of vertical FWNTs between 750 to $850{ }^{\circ} \mathrm{C}$ under our growth conditions. On silicon wafers, only short FWNT forests were observed with the height of $\sim 10 \mu \mathrm{m}$ at $850{ }^{\circ} \mathrm{C}$. These results clearly demonstrate that the quartz substrate offers unique properties that make the nucleation and growth of vertical FWNT forests more favorable.

Therefore, we investigated the catalyst distribution on the three types of substrates to further understand the effects of the substrates. Interestingly, we found that substrate types greatly 

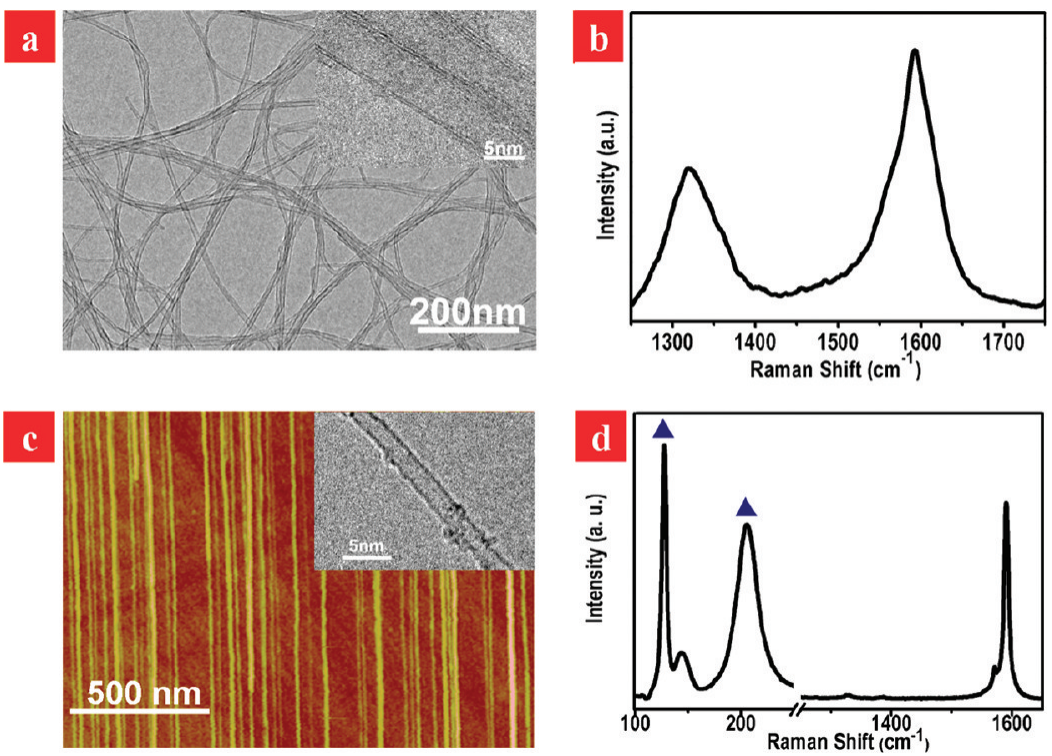

Figure 3. (a) TEM and HRTEM images of vertical forests of carbon nanotubes dispersed in methanol by ultrasonics and transferred onto a copper grid. HRTEM image shows vertical forests of carbon nanotubes that are made of FWNTs with 2-4 walls. (b) Raman spectrum of carbon nanotube forests on quartz wafers. (c) AFM image and (d) Raman spectrum of horizontal arrays of carbon nanotube on quartz wafers. The inset of (c) is a typical HRTEM image of a horizontal nanotube, after horizontal nanotube arrays have been transferred onto a copper grid from the quartz wafers. The results clearly show that horizontal arrays of carbon nanotubes are made of SWNTs. The excitation wavelength of the micro-Raman laser is $633 \mathrm{~nm}$. The peaks marked by blue triangles in the Raman spectrum arise from the single-crystal quartz substrate.

Scheme 1. Schematic Illustration of the Procedures for Orthogonally Directed Growth of Vertical FWNT Forest and Horizontal SWNT Array on Quartz Wafers

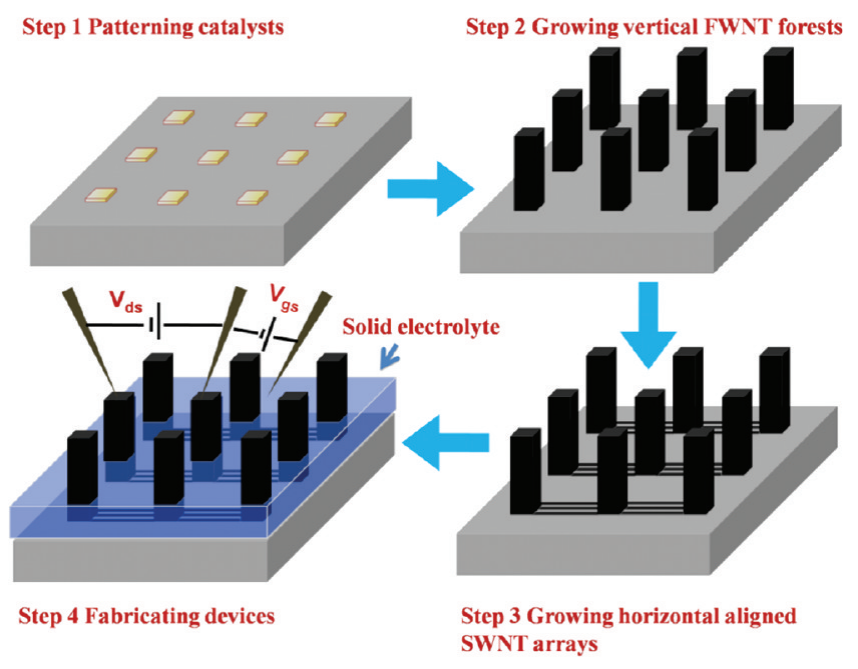

influenced the diameter distributions and density of catalyst particles even though the exact same catalyst precursors were used. Figure 5 shows AFM images of catalyst particles on quartz, sapphire, and silicon wafers after annealing the wafers coated with Shipley PR containing $2 \mathrm{mM} \mathrm{FeCl}_{3}$ at $700{ }^{\circ} \mathrm{C}$ in air for $10 \mathrm{~min}$. Catalyst particles on quartz and sapphire surfaces both show narrow distributions of diameters $(5.9 \pm 1.5 \mathrm{~nm}$ and $5.3 \pm 1.4 \mathrm{~nm}$ ), while catalyst particles on silicon surface with the diameter distribution of $9.2 \pm 2.9 \mathrm{~nm}$ show an obvious aggregation trend, indicating single-crystal surfaces have stronger interactions with catalyst particles during a heat treatment. However, on sapphire surfaces the density of particles $(\sim 260$ particles $\left./ \mu \mathrm{m}^{2}\right)$ is much lower than that on quartz wafers $(\sim 460$ particles $/ \mu \mathrm{m}^{2}$ ), which probably is the reason for the inability to obtain nanotube forests on a sapphire surface. The high density and uniform size of catalyst particles on quartz wafers are the reasons for the highly efficient growth of nanotubes. However, our experimental results do not rule out the possibility of directly growing high nanotube forests on sapphire and silicon surfaces. We believe the growth conditions may be much narrower and more critical in comparison with those for quartz substrates.

Mechanism of Orthogonal Growth of Nanotubes on Quartz Wafers. We noticed that our results supported the 'basegrowth' mechanism for the growth of FWNT forests. Before growing the horizontal SWNTs, we did not add any new catalysts, but were able to grow SWNTs from the bottom of nanotube forests, which is a strong proof for base growth mechanism. In order to further confirm this mechanism, we even removed the vertical forest islands by scraping the surface of quartz wafers after the first CVD growth, and then did the second growth. We still found many SWNTs in the area of catalyst islands (Figure 4S in the Supporting Information).

Electrical Characteristics of 3D Nanotube Structures. Since the vertical FWNT forest and the horizontal SWNT arrays are from the same catalyst pattern, they are electrically connected. The vertical forest can be used as electric contact for all-carbon FET structures. Figure 6a is the output $\left(I_{\mathrm{d}}\right.$ versus $\left.V_{\mathrm{d}}\right)$ characteristics of SWNT arrays between two FWNT forest walls without adding gate voltage. The length of the channel is $100 \mu \mathrm{m}$ and the width is $5 \mathrm{~mm}$, which includes over 3000 SWNTs. The output current of $\sim 2 \mathrm{~mA}$ verifies the good contact between vertical FWNTs and horizontal SWNTs. So we fabricated all-nanotube thin film FET devices based on this structure. The fabrication process is quite simple, and no postgrowth process is required. Similar to our previous work, ${ }^{31,36}$ we used a solid polymer electrolyte as a top gate which is made of a poly(ethylene oxide)/lithium perchlorate mixture. The overall device can be made by dropping and drying methanol solution of the polymer onto as-grown 3D nanotube structures. Figure $6 \mathrm{~b}$ shows the transfer characteristics $\left(I_{\mathrm{d}}\right.$ versus

(36) Lu, C. G.; Fu, Q.; Huang, S. M.; Liu, J. Nano Lett. 2004, 4, 623-627. 

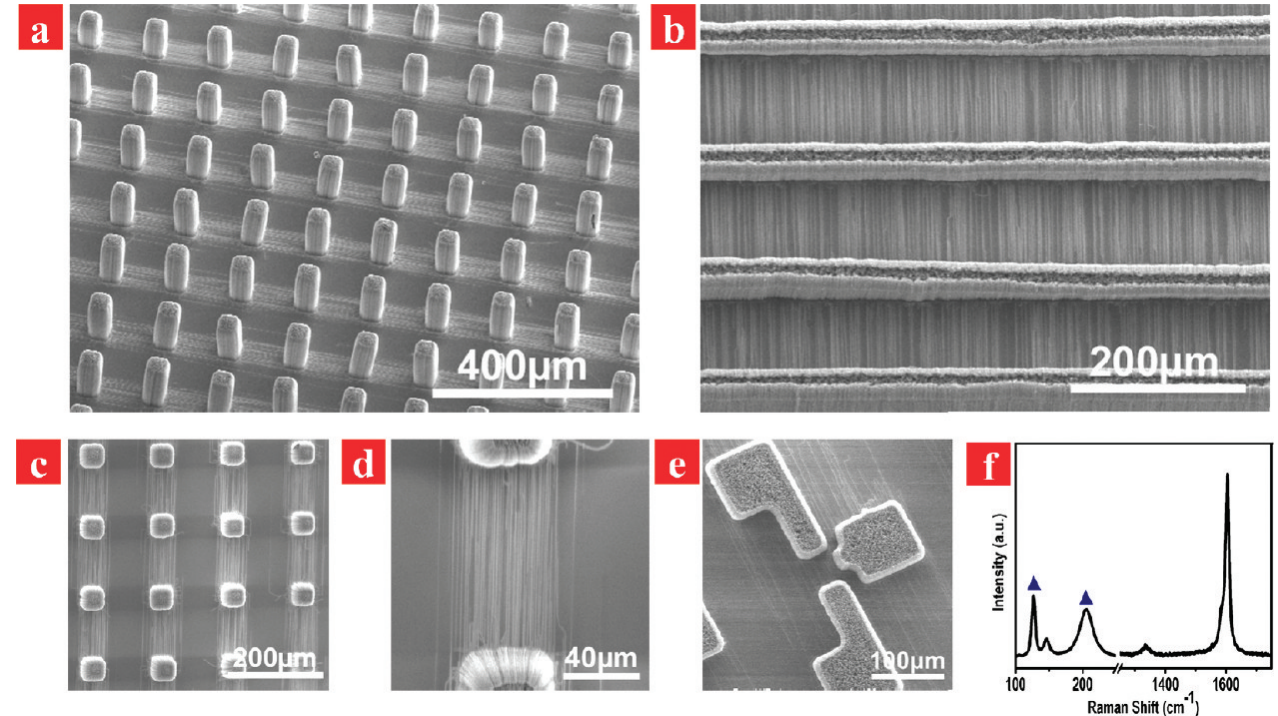

Figure 4. SEM images (a-e) of 3D CNT architectures on quartz wafers by an orthogonally directed CVD growth; typical Raman spectrum (f) of horizontal SWNTs connecting between FWNT forest islands. The excitation wavelength of the micro-Raman laser is $633 \mathrm{~nm}$. The peaks marked by blue triangles in the Raman spectrum (f) arise from the single-crystal quartz substrate. (a) SEM image of side view. (b-e) SEM images of the top view. (f) Raman spectrum of surface nanotubes.
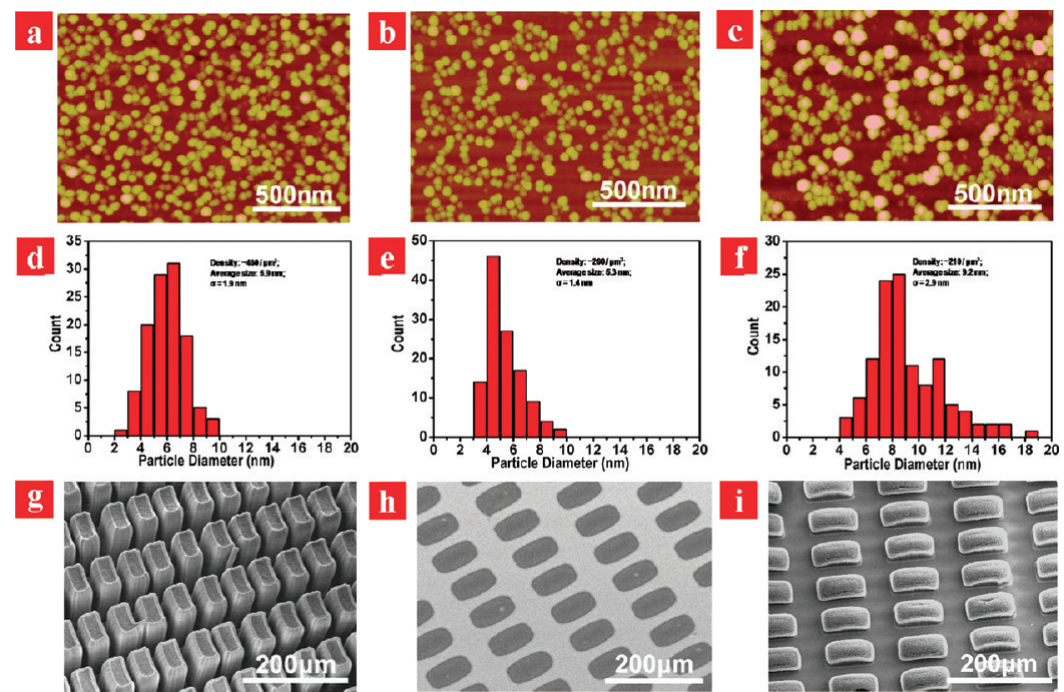

Figure 5. AFM images and size distributions of catalyst particles on the surface of quartz (a, d), sapphire (b, e), and silicon (c, f) obtained after annealing wafers with Shipley PR doped with $2 \mathrm{mM} \mathrm{FeCl}_{3}$ at $700{ }^{\circ} \mathrm{C}$ in air for $10 \mathrm{~min}$, and SEM images of the surfaces of same quartz (g), sapphire (h), and silicon (i) substrates after a CVD growth at $850{ }^{\circ} \mathrm{C}$ by water-assisted ethylene CVD. The number of counted particles on each substrate is 120.
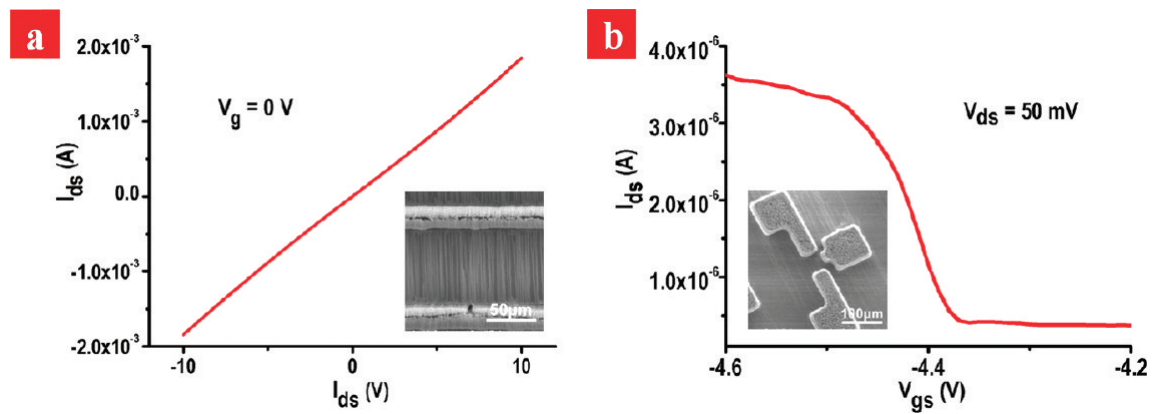

Figure 6. Output characteristics (a) of SWNT arrays between two forest walls CNT without adding gate voltage; transfer characteristics (b) of a typical FET device with the channel length and width of 50 and $40 \mu \mathrm{m}$, respectively. The bias voltage used in (b) for the measurement was $50 \mathrm{mV}$. Insets are the corresponding SEM images of the devices.

$V_{\mathrm{g}}$ ) of a typical FET device based on the nanotube structure. The length and width of the channel measured in our experiment are 50 and $40 \mu \mathrm{m}$, respectively, and the device contains $\sim 30$ SWNTs.
This polymer gating structure shows high gate efficiencies and ON/ OFF ratios $>10$, indicating a high percentage of semiconducting nanotubes similar to that in previous results. ${ }^{31} \mathrm{We}$ believe the 
performance of the devices can be further improved by decreasing the channel length.

\section{Conclusions}

In conclusion, we developed a method for orthogonal and anisotropic growth of nanotubes on $Y$-cut quartz surface. Vertically aligned FWNTs in the $Z$ direction or horizontally aligned SWNTs in the $X-Y$ plane can be selectively synthesized by delicately controlling CVD conditions. Furthermore, we have succeeded in building a 3D nanotube architecture including vertical FWNT forests and horizontal SWNT arrays on quartz. Our experimental results demonstrate quartz wafers can not only guide the growth of horizontal aligned SWNT arrays but are also suitable for the growth of vertical FWNT forests. The main reason is that on quartz surface, relatively high density and uniform catalyst particles were formed comparing with using sapphire and silicon as substrates. We further comments on 'base-growth' as the growth mechanism of nanotube forests.
FET devices based on this 3D nanotube architecture were fabricated and measured using a solid polymer as top gating structure. The applications of this device in gas sensors or electrochemical sensors are very promising since it will rule out the contributions from metal electrodes. In addition, this complex structure can be synthesized at wafer scale. We believe our approach opened a new window for constructing a more complex 'nanotube world' on chips.

Acknowledgment. The work is supported in part by a grant from NRL (N00173-04-1-G902) and ONR (N00014-09-1-0163). We also acknowledge the Shared Materials Instrumentation Facility (SMIF) at Duke University for access to their instrumentation.

Supporting Information Available: Further experimental details and methods, supportive SEM data. This material is available free of charge via the Internet at http://pubs.acs.org.

JA908414V 